\title{
A Case Of Awake Percuitaneous Extracorporeal Membrane Oxygenation For High-risk Percutaneous Coronary Intervention
}

\author{
Shuangbo Liu ${ }^{1}$, Amir Ravandi ${ }^{2}$, Malek Kass ${ }^{3}$, Basem Elbarouni ${ }^{4}$ \\ 1. Cardiology, St. Michael's Hospital, Toronto, CAN 2. Section of Cardiology, St. Boniface Hospital, \\ University of Manitoba, Canada 3. Cardiology, University of Manitoba, Winnipeg, CAN 4. Section of \\ Cardiology, Department of Internal Medicine, University of Manitoba, Canada
}

$\square$ Corresponding author: Shuangbo Liu, shuangboliu@gmail.com

Disclosures can be found in Additional Information at the end of the article

\section{Abstract}

With significant improvements in percutaneous coronary intervention (PCI) technology, complex high risk PCI is increasingly offered to patients with limited revascularization options. Percutaneous mechanical circulatory support devices are often utilized for hemodynamic support during these complex procedures. Veno-arterial extracorporeal membrane oxygenation (ECMO) allows full hemodynamic support and provides systemic oxygenation. We describe a case of left main bifurcation stenting performed at our center with ECMO support in an awake patient without general anesthesia.

Categories: Cardiology

Keywords: ecmo, pci, venoarterial extracorporeal membrane oxygenation (va-ecmo), percutaneous coronary intervention

\section{Introduction}

Significant improvements in percutaneous coronary intervention (PCI) technology has led to complex high-risk PCI being offered to patients with limited revascularization options. Percutaneous mechanical circulatory support (MCS) devices, including veno-arterial extracorporeal membrane oxygenation (VA-ECMO), are often utilized for hemodynamic support during these complex procedures. VA-ECMO provides cardiopulmonary support and is comprised of a venous cannula to drain the deoxygenated blood, centrifugal nonpulsatile pump, membrane for gas exchange and an arterial cannula to return the oxygenated blood to the body [1]. This allows for full hemodynamic support, with cardiac outputs over $4.5 \mathrm{~L} / \mathrm{min}$ [2].

Received 03/14/2017

Review began 03/15/2017

Review ended 04/24/2017

Published 04/25/2017

C Copyright 2017

Liu et al. This is an open access article distributed under the terms of the Creative Commons Attribution License CC-BY 3.0., which permits unrestricted use, distribution, and reproduction in any medium, provided the original author and source are credited.
The definition of high-risk PCI is currently evolving. Patient, lesion and clinical presentation risk factors need to be considered when making the decision about need for percutaneous hemodynamic support [1]. Commonly considered factors include increased age, reduced left ventricular systolic function, ACC/AHA Type C lesions, vessels that supply a large area (unprotected left main, last patent vessel or severe 3 vessel disease) or acutely ill patients (large myocardial infarction or cardiogenic shock) [1]. No randomized clinical trial exists to assess the use of ECMO in high-risk PCI.

\section{Case Presentation}

How to cite this article

Liu S, Ravandi A, Kass M, et al. (April 25, 2017) A Case Of Awake Percutaneous Extracorporeal

Membrane Oxygenation For High-risk Percutaneous Coronary Intervention. Cureus 9(4): e1191. DOI 10.7759/cureus. 1191 


\section{Cureus}

A 54-year old male with a history of ischemic heart disease and hypertension presented with an acute coronary syndrome (ACS). Coronary angiography demonstrated a left dominant system with a $90 \%$ stenosis in the distal left main (LM) extending into the left circumflex (Cx) and left anterior descending (LAD) arteries, in addition to a previously documented $100 \%$ mid-LAD occlusion (Figure 1A, 1B). Left ventricular (LV) ejection fraction was estimated at 30-40\% by transthoracic echocardiogram. He was not a surgical candidate given the lack of graftable targets. The patient expressed wished to pursue revascularization due to ongoing symptoms. We anticipated that wiring the $\mathrm{Cx}$ would be challenging due to an acute takeoff angle with a high risk of acute vessel closure. Given the reduced LV systolic function as well as complex anatomy, we discussed the need for percutaneous MCS. In discussion with our Heart Team, we proceeded with high-risk PCI with awake ECMO support.

The patient was lightly sedated with midazolam and fentanyl. PCI was performed via the right femoral artery using a 7F sheath. ECMO cannulation (Figure 1C) was via the left femoral artery (17F) and right femoral vein (24/29F 2-stage venous cannula). We pre-closed both access sites with two Perclose ProGlide (Abbott Vascular, Redwood City, CA) placed at orthogonal angles of 10 and 2 o'clock. ECMO flow was set at $3.5 \mathrm{~L} / \mathrm{min}$ with $3360 \mathrm{RPM}$ using a BioMedicus 540 centrifugal pump (Medtronic, Minneapolis, MN). A SuperCross micro-catheter with a preformed 1200 angled tip (Vascular Solutions, Minneapolis, MN) was used and the Cx was wired using a Sion wire (ASAHI, Abbott vascular, Redwood City, CA). PCI was performed with excellent results using a T-and protrusion (TAP) bifurcation technique with two drug-eluting stents (Figure 1D). The patient was hemodynamically stable throughout the procedure with no chest pain despite prolonged and high-pressure inflations in the LM. After PCI, the patient was weaned off ECMO and decannulated successfully (Figure $1 E$ ) in the cath lab. To maintain vascular access, we punctured and passed a $260 \mathrm{~cm}$ J wire through both ECMO cannulas before removing them (Figure 1F). A third Perclose at 12 o'clock was needed to achieve hemostasis in both access sites. The patient was transferred to the ward, discharged two days later, and was doing well at one month follow up.

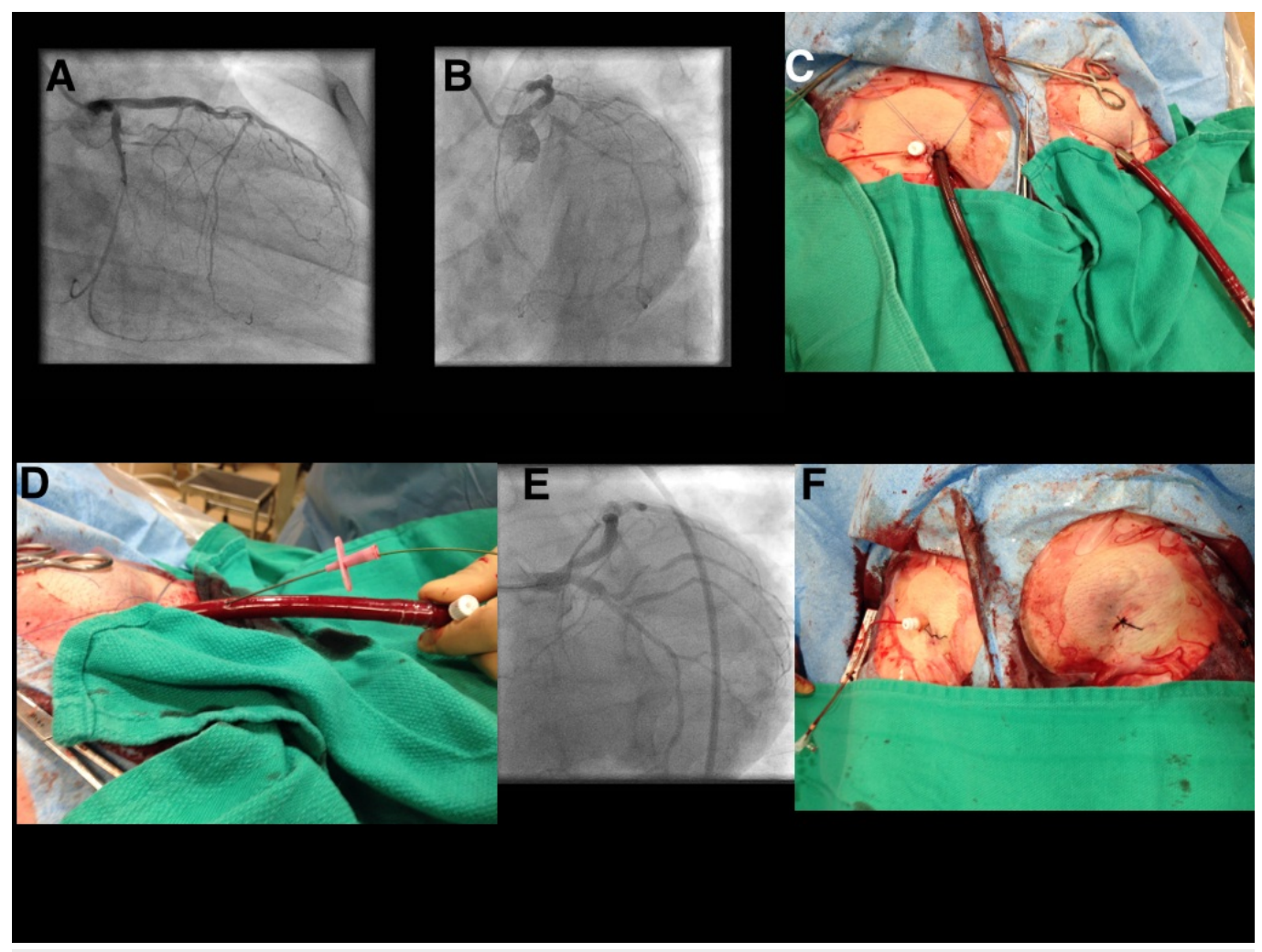




\section{FIGURE 1: Case illustration}

Figure 1A: Pre-procedure coronary angiography (AP caudal projection) demonstrating severe distal left main stenosis involving left main bifurcation

Figure 1B: Pre-procedure coronary angiography (left anterior oblique caudal projection) demonstrating severe distal left main stenosis involving left main bifurcation

Figure 1C: Intra-procedure image demonstrating arterial and venous cannulation

Figure 1D: Fluoroscopy image (left anterior oblique caudal projection) demonstrating the left coronary system after successful PCl

Figure 1E: End of procedure image demonstrating arterial cannula puncture and wiring to maintain vascular access

Figure 1F: After successful ECMO decannulation and hemostasis

\section{Discussion}

With significant improvements in PCI technology, complex high-risk PCI is increasingly offered to patients with limited revascularization options. Percutaneous MCS devices are often utilized for hemodynamic support during these complex procedures.

IABP is the most common type of MCS used during PCI, mainly due to its ease of use. A single double lumen catheter with a balloon at the end is inserted and attached to a pump. Helium inflates the balloon at the beginning of diastole. IABP increases diastolic blood pressure and coronary artery perfusion decreases afterload and myocardial oxygen consumption and may increase cardiac output slightly [1]. Advantages include being available in most centers and ease of use. A disadvantage of IABP is the need for a stable cardiac rhythm and not being able to provide full hemodynamic support.

Another type of MCS is the Impella (Abiomed, Danvers, Massachusetts), which is a device placed across the aortic valve. When optimally positioned, it propels blood from the LV to the aorta. The Impella decreases end-diastolic wall stress while increasing diastolic compliance as well as aortic and intracoronary pressures and coronary flow velocity reserve [1]. Advantages include enhanced cardiac output (2.5-4 L/min, depending on cannula size) and does not require a stable cardiac rhythm to function. However, it may not be universally available, requires larger cannula size and therefore higher risk of complications compared to IABP.

While traditionally, IABP and Impella are used, the former might not provide adequate hemodynamic support [1] and the latter can be quite expensive. VA-ECMO allows full hemodynamic support and provides systemic oxygenation [2]. Other advantages include ease of use, the ability to augment cardiac output by $>3.5 \mathrm{~L} /$ minute and unlike IABP, does not require a stable cardiac rhythm for optimal function [1]. It also provides better circulatory support at a reduced cost in comparison to Impella. Limitations include local expertise, the need for a larger arterial cannula, and the need to cannulate the femoral vein. Post-procedural care of ECMO patients can be challenging and resource intensive, but as demonstrated in our case, avoiding intubation and general anesthesia by performing awake ECMO with successful percutaneous closure and hemostasis allows for rapid mobilization and patient discharge. This is the second case of awake ECMO performed at our center; both with a favorable outcome and successful percutaneous hemostasis [3]. Both cases demonstrate the safety and feasibility of awake ECMO in high-risk PCI procedures. 


\section{Conclusions}

Percutaneous mechanical circulatory support devices, including awake VA-ECMO, can be safely performed in appropriate cases of high-risk PCI.

\section{Additional Information \\ Disclosures}

Human subjects: Consent was obtained by all participants in this study. Conflicts of interest: In compliance with the ICMJE uniform disclosure form, all authors declare the following:

Payment/services info: All authors have declared that no financial support was received from any organization for the submitted work. Financial relationships: All authors have declared that they have no financial relationships at present or within the previous three years with any organizations that might have an interest in the submitted work. Other relationships: All authors have declared that there are no other relationships or activities that could appear to have influenced the submitted work.

\section{References}

1. Rihal CS, Naidu SS, Givertz MM, Szeto WY, Burke JA, Kapur NK et al; : 2015 SCAI/ACC/HFSA/STS Clinical expert consensus statement on the use of percutaneous mechanical circulatory support devices in cardiovascular care. J Am Coll Cardiol. 2015, 65(19):e7-e26. 10.1016/j.jacc.2015.03.036

2. Myat A, Patel N, Tehrani S, Banning AP, Redwood SR and Bhatt DL: Percutaneous circulatory assist devices for high-risk coronary intervention. JACC: Cardiovasc Interv. 2015, 8(2):229-44. 10.1016/j.jcin.2014.07.030

3. Kass M, Moon M, Vo M, Ravandi A: Awake extracorporeal membrane oxygenation for very high-risk coronary angioplasty . Can J Cardiol. 2015, 31(2):227:e11-13.

10.1016/j.cjca.2014.11.004 